\title{
Study on the Bonding Mechanism and Bonding Performance of Double Layer Fine-Surfacing Technology
}

\author{
Xiangyan $\mathrm{Wu}$, Xiangling $\mathrm{CHEN}^{1}$, Jianping YANG, Xin HU and Guang YANG \\ Chongqing Chengbang Pavement Material Co., Ltd, Chongqing, China
}

\begin{abstract}
Double layer fine-surfacing technology is a thin layer maintenance technology of pavement positive texture, which plays an important role in preventing road safety accidents. Bonding performance is the key quality control index of the double layer fine-surfacing, which directly affects its service performance and durability. In this paper, the bonding mechanism of the double layer fine-surfacing is analyzed, and the bonding strength between the double layer fine-surfacing and the old asphalt pavement surface is studied by using the pullout test method under the factors of different cement dosage and different temperature environment, and the bonding index of the double layer fine-surfacing is put forward. Experiments show that the double layer fine-surfacing technology has the characteristics of super bonding and wear resistance. It is an economical and practical maintenance technology and has broad development prospects.
\end{abstract}

Keywords. Double layer fine-surfacing technology, road safety, bonding performance

\section{Introduction}

Under the influence of long-term traffic load and climate environment, asphalt pavement will suffer from diseases such as asphalt aging, cracks, looseness, and insufficient antisliding performance after being put into operation for a period. Road performance will gradually decline, service ability will gradually decline, and road maintenance and traffic safety problems will become increasingly prominent. It is particularly important to take different maintenance measures to protect the pavement at different stages of the whole life cycle.

At present, the commonly used surface maintenance technologies of pavement include micro-surfacing, slurry seal, thin-layer overlay and so on. The cementing materials used in this kind of technology are mainly emulsified asphalt or modified emulsified asphalt. Affected by ambient temperature, hydrodynamic pressure and inherent defects of the product itself, there are generally problems such as insufficient adhesion, poor durability, easy peeling, poor environmental protection, and high construction requirements. The use of these technologies is limited and cannot meet the market demand. Compared with the traditional maintenance technology, the double layer precision surface treatment technology has the characteristics of super-strong

1 Corresponding Author, Xiangling CHEN, Chongqing Chengbang Pavement Material Co., Ltd, Chongqing, China; Email: 383114392@qq.com. 
bonding, high anti-sliding, and durable wear resistance, which can improve the antisliding and waterproof performance of the pavement and prolong the service life of the road. Its high anti-sliding performance and special texture structure can prevent vehicle slippage and rollover, effectively improve traffic safety, and reduce the traffic accident rate by more than $70 \%$. It is of great significance and role to solve the active safety problem of highways in China and reduce the traffic accident rate.

In the construction process of double layer fine-surfacing, the bonding forming mechanism and bonding performance directly affect the construction technology, construction quality and road performance of fine-surfacing, and directly determine its service life. In this paper, through the analysis of the bonding forming mechanism of the fine-surfacing and the study of the interlayer bonding performance, the bonding strength requirements of the double layer fine-surfacing are proposed, to effectively guide the engineering quality of the fine-surfacing.

\section{Introduction of Double Layer Fine-Surfacing Technology}

Double layer fine-surfacing is under the condition of good road traffic quality, using the special construction equipment of fine-surfacing, epoxy composite modified asphalt, aggregate, high viscosity emulsified asphalt is spread to the asphalt pavement layer by layer, and a layer of ultra-thin, wear-resistant, anti-skid and waterproof protective layer is formed on the road surface quickly by appropriate rolling [1-5]. As a pavement surface maintenance technology, the thickness of the fine-surfacing construction is generally 3$8 \mathrm{~mm}$ according to the model, which is mainly used for the prevention and maintenance of highway and urban road asphalt pavement surface and the recovery and improvement of asphalt pavement skid resistance.

In order to ensure that the double layer fine-surfacing has sufficient structural strength, a typical sandwich structure is adopted. There are five layers from bottom to top. The specific structural schematic diagram is shown in figure 1.

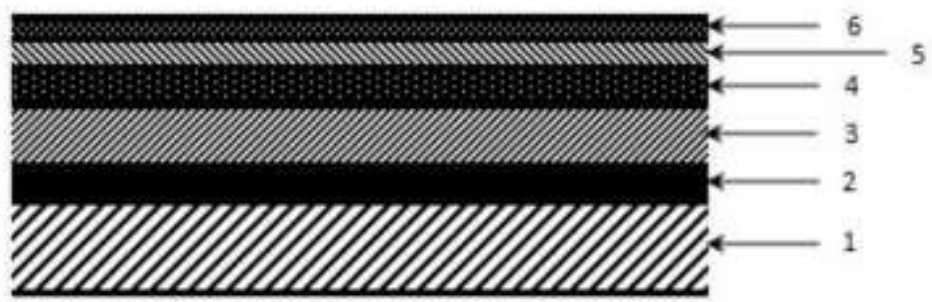

(1 - original pavement. 2 - First layer: epoxy composite modified asphalt. 3-Second layer: aggregate. 4-The third layer: high viscosity emulsified asphalt. 5-Fourth layer: aggregate. Layer 6 - 5: high viscosity emulsified asphalt)

Figure 1. Diagram of pavement structure for double layer fine-surfacing.

The double layer fine-surfacing is mainly constructed by three processes [6, 7]. The specific construction technology is:

(1) Investigate and deal with the original pavement diseases in advance, and prepare the relevant people, machines, and materials.

(2) On-site traffic control, road cleaning and marking removal. 
(3) Construction of the first process: the epoxy composite modified asphalt and aggregate are simultaneously sprinkled onto the original pavement by a dedicated sprinkling vehicle for maintenance and molding.

(4) Construction in the second process: after drying and molding in the first process, high-viscosity emulsified asphalt and aggregate were synchronously spread onto the first material by using a special spraying vehicle. After the material is demulsification, the roller is used for rolling to make it compact.

(5) Construction of the third process: spraying high viscosity emulsified asphalt on the first and second materials. After the material is demulsification, the roller is used for rolling to ensure compactness and smoothness.

(6) Maintenance molding, after the formation of strength, speed limit open traffic.

\section{Analysis on Bonding Forming Mechanism of Double Layer Fine-Surfacing}

Bonding is the result of interaction between different material interfaces after contact. The bonding of double layer fine-surfacing mainly includes two aspects: one is the bonding between the structural layer of double layer fine-surfacing and the original pavement. The other is the bonding between aggregate and binder in the structural layer. The former is mainly affected by the performance of epoxy composite modified asphalt and the technical status of the original pavement, while the latter is mainly affected by the performance of high-viscosity emulsified asphalt, the compaction between the upper and lower aggregates, and the rolling compaction $[8,9]$.

Combined with the structure design and construction technology of double layer fine-surfacing, the bond strength formation of fine-surfacing mainly includes the following two stages:

\subsection{Formation of Bond Strength in the First Process}

Epoxy composite modified asphalt used in double layer fine-surfacing is an oil-soluble composite material with good fluidity and adhesion. The aggregate used is basalt stone. After the epoxy composite modified asphalt is constructed on the pavement, it can quickly penetrate the pavement surface through the micro cracks and pores in the original pavement structure, continuously penetrate and adsorb the contact asphalt mixture, and bring the active substances in the epoxy composite modified asphalt to the depth of the pavement surface. On the one hand, the active substances penetrate the original pavement continuously repair the aging tissue. on the other hand, cross-linking reactions such as interfacial tension, surface free energy and functional group properties occur with the adhesive to enhance the bonding between the double layer fine-surfacing and the original pavement. Aggregates distributed synchronously form skeletons with each other by embedded extrusion force and internal friction resistance, and are in close contact with epoxy composite modified asphalt. Under the action of pore adsorption on aggregate surface, self-weight and temperature environment, aggregate and epoxy composite modified asphalt are gradually bonded together. At the same time, a series of crosslinking reactions of epoxy, asphalt and other substances in epoxy composite modified asphalt further accelerate the bonding between epoxy composite modified asphalt, aggregate and the original pavement, so that it is finally consolidated into a whole. With the extension of curing time, the bonding strength gradually forms, which provides a prerequisite for the overall bonding of the subsequent process layer. The bond strength 
at this stage is mainly formed by the penetration and adsorption of epoxy composite modified asphalt, cross-linking reaction and the interlocking of aggregate.

\subsection{Formation of Bond Strength in Second and Third Processes}

The high viscosity emulsified asphalt used in the double layer fine-surfacing is a kind of high viscosity polymer modified emulsified asphalt, belonging to the cationic type, and the aggregate used is basalt stone. The second and third processes are constructed in the form of high viscosity emulsified asphalt, aggregate, and high viscosity emulsified asphalt. It can be said that the asphalt is fully distributed, and it is easier to stabilize and form under rolling. The high viscosity emulsified asphalt has a positive charge and the aggregate has a negative charge [10]. After the two are synchronously constructed on the pavement, the high viscosity emulsified asphalt adsorbs the upper and lower layers of aggregate surface at the same time, and gradually occurs water evaporation, demulsification, condensation and solidification reactions, forming a preliminary bond strength. After demulsification, the high viscosity emulsified asphalt is rolled by roller. Under the action of rolling, the aggregate is further compacted, and the strength is formed by the interlocking force and internal friction resistance. At the same time, the high viscosity emulsified asphalt climbs to fill the gap between the aggregates, so that the aggregate and the high viscosity emulsified asphalt form a whole, and the bonding strength is further enhanced. In this stage, the bonding strength at the fine-surfacing is mainly formed by the charge adsorption between high-viscosity emulsified asphalt and aggregate, the demulsification of binder, vehicle rolling, and the inlay and extrusion between aggregates.

After opening the traffic, the double layer fine-surfacing is continuously rolled by the vehicle load, and the moisture in the residual high-viscosity emulsified asphalt in the structural layer is further evaporated. The contact between the aggregate and the aggregate, and between the aggregate and the high-viscosity emulsified asphalt is closer. The bond strength between the double layer fine-surfacing and the original pavement is also gradually increased. According to different climate environments, it generally reaches the maximum in about 7 days and tends to be stable. At this stage, the formation of fine-surfacing bond strength is mainly contributed by the rolling effect of social vehicles.

\section{Evaluation of Bond Strength}

According to the analysis of the bonding forming mechanism of the double layer finesurfacing, the bonding between the first epoxy composite modified asphalt and the original pavement plays a key role in the bonding contribution of the whole structure. If the interlayer bonding ability is insufficient, it is prone to peeling, insufficient adhesion and poor durability, which directly affects the quality of double layer fine watch.

In order to characterize the Bonding properties of epoxy composite modified asphalt on the original pavement, the author simulated the actual road conditions of the pavement, and used the special modified asphalt to form the AC-13 asphalt mixture rutting plate in the room, and formed the specimen in the upper part according to the first process at the double fine-surfacing. The epoxy composite modified asphalt selected the KP-G13 high performance cement produced by Chongqing Chengbang Pavement Materials Co., Ltd., and the aggregate selected basalt stone with particle size of 1-3 $\mathrm{mm}$. The bonding 
strength of the first layer epoxy composite modified asphalt on the original pavement under different binder content and temperature environment was systematically studied by interlayer drawing test.

\subsection{Bond Strength under Different Binder Dosages}

The amount of binder has a certain influence on the bonding performance of epoxy composite modified asphalt and original pavement. In this paper, the bond strength of epoxy composite modified asphalt to rutting plate under different binder dosages is evaluated. The results are shown in table 1 and figure 2 .

Table 1. Bond strength of epoxy composite modified asphalt to rutting plate without binder content.

\begin{tabular}{ll}
\hline Binder content $\left(\mathbf{k g} / \mathbf{m}^{\mathbf{2}}\right)$ & Average bond strength $\mathbf{( M P a )}$ \\
\hline 0.3 & 2.26 \\
0.4 & 3.14 \\
0.6 & 2.76 \\
0.8 & 2.13 \\
1.0 & 1.99 \\
1.2 & 1.96 \\
\hline
\end{tabular}

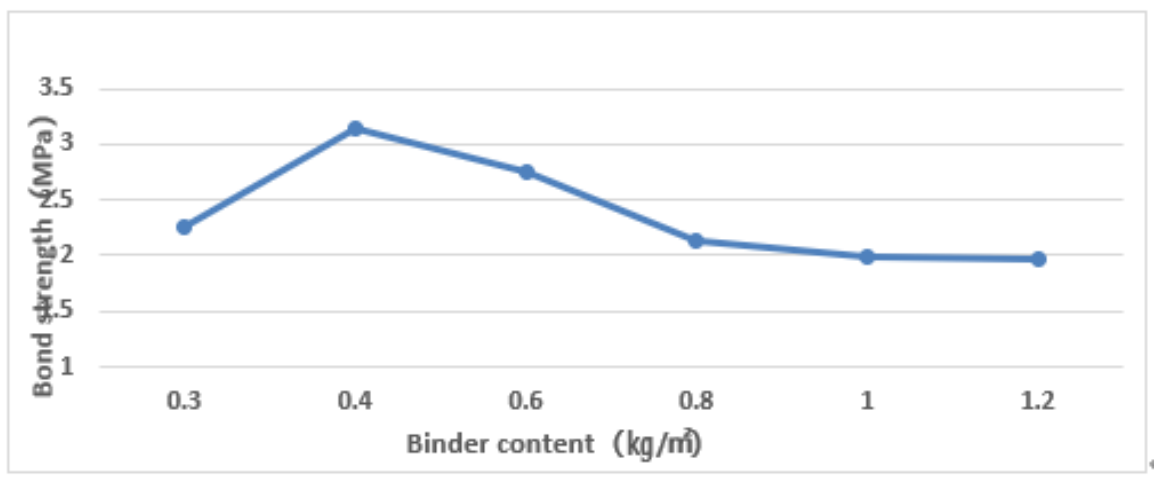

Figure 2. Effect of binder content on bond strength.

From the above tests, it can be found that under the same road technical conditions, the amount of cement increased from $0.3 \mathrm{~kg} / \mathrm{m}^{2}$ to $0.4 \mathrm{~kg} / \mathrm{m}^{2}$, and the bond strength of epoxy composite modified asphalt on rutting plate increased from $2.26 \mathrm{MPa}$ to $3.14 \mathrm{MPa}$. When the binder content increased from $0.4 \mathrm{~kg} / \mathrm{m}^{2}$ to $1.2 \mathrm{~kg} / \mathrm{m}^{2}$, the bond strength of epoxy composite modified asphalt on rutting plate decreased from the maximum of 3.14 $\mathrm{MPa}$ to the minimum of $1.96 \mathrm{MPa}$. When the binder content increased from $0.4 \mathrm{~kg} / \mathrm{m}^{2}$ to $0.8 \mathrm{~kg} / \mathrm{m}^{2}$, the bond strength attenuation was the largest, reaching $31.8 \%$, and the epoxy adhesive layer of the failure section gradually transited to the binder layer. When the cement content increased from $0.8 \mathrm{~kg} / \mathrm{m}^{2}$ to $1.2 \mathrm{~kg} / \mathrm{m}^{2}$, the attenuation of bond strength was relatively flat, with a decrease of only $8 \%$, and the failure section was in the cement layer. Through the test under different binder dosages, when the binder dosage is 0.3 $\mathrm{kg} / \mathrm{m}^{2}$, the epoxy composite modified asphalt wrapped between the rutting plate and the aggregate is less, and the thickness of the adhesive layer is thin, which makes the epoxy composite modified asphalt insufficiently bonded to the rutting plate and the aggregate. 
When the binder dosage increases to $0.4 \mathrm{~kg} / \mathrm{m}^{2}$, the epoxy composite modified asphalt wrapped between the rutting plate and the aggregate is appropriate, and the thickness of the adhesive layer is appropriate, and the interlayer bonding strength reaches the maximum. When the binder content increases again, the epoxy composite modified asphalt wrapped between the rutting plate and the aggregate is too much, and the thickness of the adhesive layer is too large. The interlayer bonding strength decreases gradually with the increase of the thickness of the adhesive layer, and is finally stable.

\subsection{Bond Strength under Different Test Temperatures}

Temperature has a certain influence on the bonding of epoxy composite modified asphalt. In this paper, the bond strength of epoxy composite modified asphalt on rut plate at $10^{\circ} \mathrm{C}$, $25^{\circ} \mathrm{C}, 40^{\circ} \mathrm{C}, 60^{\circ} \mathrm{C}$ and $80^{\circ} \mathrm{C}$ is evaluated. The results are shown in table 2 and figure 3 .

Table 2. Bond strength of epoxy composite modified asphalt to rut plate under different test temperatures.

\begin{tabular}{ll}
\hline Test temperature $\left({ }^{\circ} \mathbf{C}\right)$ & Average bond strength $(\mathbf{M P a})$ \\
\hline 10 & 2.76 \\
25 & 1.96 \\
40 & 1.30 \\
60 & 0.76 \\
80 & 0.62 \\
\hline
\end{tabular}

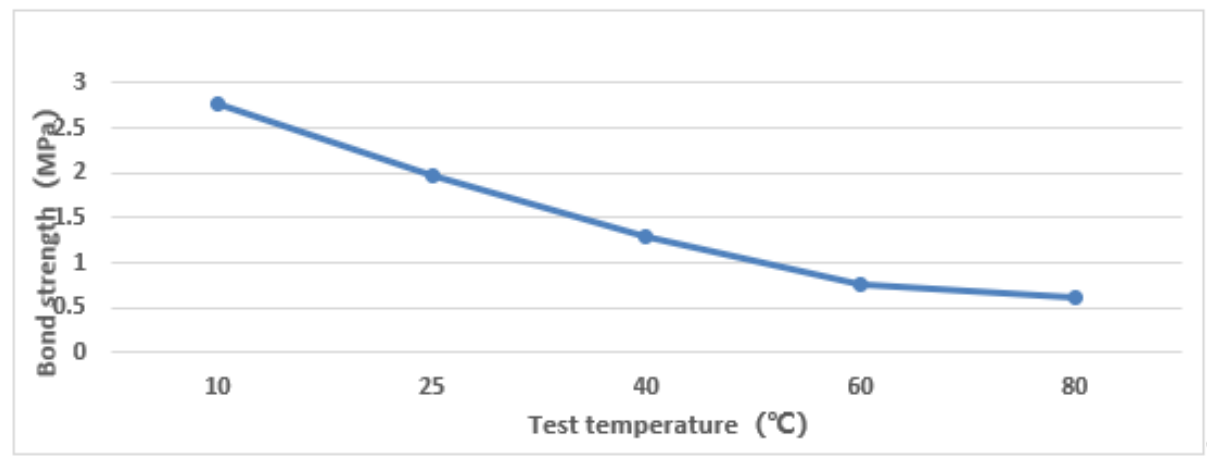

Figure 3. Effect of test temperature on bond strength of epoxy composite modified asphalt for rutting plate.

From table 2, it is found that when the test temperature rises from $10{ }^{\circ} \mathrm{C}$ to $60{ }^{\circ} \mathrm{C}$, with the increase of temperature, the bond strength decreases linearly, from $2.76 \mathrm{MPa}$ to $0.76 \mathrm{MPa}$, and the decrease is $72.5 \%$. When the temperature increased from $60{ }^{\circ} \mathrm{C}$ to $80{ }^{\circ} \mathrm{C}$, the bond strength decreased slowly, from $0.76 \mathrm{MPa}$ to $0.62 \mathrm{MPa}$, with a decrease of only $18.4 \%$. It can be seen from this group of tests that the temperature sensitivity of epoxy composite modified asphalt is relatively sensitive, and the test temperature has a great influence on the bond strength of epoxy composite modified asphalt. In road engineering, $25^{\circ} \mathrm{C}$ and $60^{\circ} \mathrm{C}$ are generally used to measure the performance of materials at room temperature and high temperature. Therefore, this paper also proposes to use $25^{\circ} \mathrm{C}$ bond strength and $60^{\circ} \mathrm{C}$ bond strength to control the quality of epoxy composite modified asphalt. According to the requirements of relevant standards for epoxy composite modified asphalt, the bond strength at $25^{\circ} \mathrm{C}$ should not be less than $1.0 \mathrm{MPa}$, and the bond strength at $60^{\circ} \mathrm{C}$ should not be less than $0.5 \mathrm{MPa}$. 


\section{Conclusions}

(1) Based on the analysis of the bonding performance between the double layer finesurfacing and the original pavement, as well as between the binder and the aggregate, the bonding strength of the double layer fine-surfacing is mainly formed by the infiltration and adsorption between the binder and the aggregate, the crosslinking reaction, the and extrusion between the aggregates, and the rolling of vehicles.

(2) The pull-out test can better characterize the bonding performance between the double layer fine-surfacing and the original pavement. Through the study of bonding performance under different binder dosage and test temperature, with the increase of binder dosage and temperature, the bonding strength of fine-surfacing shows a downward trend. It is proposed that the bonding strength at $25{ }^{\circ} \mathrm{C}$ is not less than 1.0 $\mathrm{MPa}$, and the bonding strength at $60^{\circ} \mathrm{C}$ is not less than $0.5 \mathrm{MPa}$, which is far more than the same surface maintenance technology, and the bonding performance between the fine-surfacing and the asphalt pavement is excellent.

(3) Double layer fine-surfacing treatment technology is an economical and practical surface maintenance technology, which has the characteristics of super-strong bonding, high anti-sliding and durable wear resistance. It enriches and improves the maintenance strategy of highway pavement in China, and is of great significance to improve the service ability of roads and prolong the service life of roads.

\section{References}

[1] Hu X, Wu X Y, Chen X L, Liu B Y and Yang J P 2020 Analysis of preventive maintenance materials for asphalt pavement surface finish Low Carbon World 10 (09) 143-144.

[2] Wu X Y, Xie D L, Yang G and Liu B Y 2016 Mechanism and construction technology of asphalt pavement fine-surfacing technology Road Construction Machinery and Construction Mechanization 26 (3) $31-34$.

[3] Wu X Y, Xie D L, Liu A and Liu B Y 2016 Application of surface finish technology in pre-maintenance of asphalt concrete bridge deck Road Construction Machinery and Construction Mechanization 33 (8) 35-38.

[4] Zhang X Q, Li W, Peng S C, Wu X Y and Hu X 2020 Application of asphalt pavement surface technology in Guizhou highway maintenance Traffic World (16) 74-79.

[5] Chen X L, Wu X Y, Hu X, Ni M and Yang J P 2019 The application of Jingbiao in highway anti-skid maintenance Low Carbon World (2) 203-204.

[6] Chen X L, Wu X Y, Huang J, Wu F and Wu Y 2020 A polymer composite modified emulsified asphalt for asphalt pavement surface maintenance technology Low Carbon World 10 (12) 197-198.

[7] Wu X Y, Xie D L, Yang J P, Hu X and Liu B Y 2018 Application of compound surface technology in urban roads Municipal Facilities Management (3) 14-16.

[8] Liu D 2013 Study on Pavement Performance and Evaluation Index System of Fiber Asphalt Macadam Sealing in Hot and Humid Areas of South China (Xi'an: Chang'an University).

[9] Li K and Li Y H 2009 The formation mechanism of bonding strength between layers of fiber seal Road Traffic Technology 10 (5) 36-46.

[10] Li J J 2018 Study on the Material and Structural Characteristics of Epoxy Anti-skid Wear Layer on Cement Pavement (Xi'an: Chang'an University). 